\title{
Comparação de Desempenho entre o Método dos Elementos de Contorno com Integração Direta e o Método dos Elementos Finitos em Problemas de Helmholtz
}

\author{
Loeffler Neto, C. F. ${ }^{*} ;$ Mansur, W.J. ${ }^{2} ;$ Barcelos, H.M. ${ }^{1^{*}}$ \\ 1 Programa de Pós-Graduação em Engenharia Mecânica, Universidade Federal do Espírito Santo, Vitória, ES, Brasil \\ 2 Programa de Pós-Graduação em Engenharia Civil, Universidade Federal do Rio de Janeiro, Rio de Janeiro, RJ, Brasil \\ * e-mail: carlosloeffler@bol.com.br \\ * e-mail: webe@coc.ufrj.br \\ *e-mail: engercules@gmail.com
}

\begin{abstract}
Resumo
Objetiva-se avaliar o desempenho do MECID (Método dos Elementos de Contorno com Interpolação Direta) para resolver o termo integral referente à inércia na Equação de Helmholtz e, deste modo, permitir a modelagem dos espectros de resposta e a solução do problema de autovalor, comparando seus resultados com os obtidos pelo MEF (Método dos Elementos Finitos). Os problemas resolvidos pertencem a importantes áreas da engenharia e física, como no eletromagnetismo e em problemas elásticos particulares. É sabido das dificuldades existentes na aproximação precisa de distribuições mais complexas de cargas e sorvedouros no interior do domínio para qualquer técnica de contorno. No entanto, este trabalho mostra que, apesar de tais dificuldades, o desempenho do MECID é bastante satisfatório, tanto no cálculo da variável básica, quanto na sua derivada. Para tanto, são resolvidos problemas bidimensionais referentes à membrana elástica com solução tipo harmônica, além da determinação das frequências naturais em problemas acústicos em domínios fechados, utilizando malhas com diferentes graus de refinamento, além de elementos lineares com funções de bases radiais para o MECID. São geradas curvas de desempenho através do cálculo do erro médio percentual para cada malha, demonstrando a convergência de cada método, e os resultados são comparados com as soluções analíticas.
\end{abstract}

\begin{abstract}
The objective is to evaluate the performance of DIBEM (Boundary Element Method with Direct interpolation), solving the integral term relative to the inertia in the Helmholtz equation and thus allow the modeling of eigenvalue problem, calculating the natural frequencies and comparing with the results obtained by the FEM (Finite Element Method). The problems approached are applied in important areas of engineering and physics, such as electromagnetism and elasticity. It is well known the difficulties for any boundary technique to approximate more complex domain actions. However, this work shows that in spite of these difficulties, the performance of DIBEM is superior to calculate of the basic variable and its normal derivative. Thus, two-dimensional elastic membranes with harmonic type solution are solved, as well the determination of natural frequencies for closed domains. Many meshes with different degrees of refinement are tested, using linear elements and radial basis functions. Performance curves are generated by calculating of the average percentage error for each mesh, showing the convergence of each method and, when possible, the data are compared with the analytical solutions available.
\end{abstract}

Keywords: Equação de Helmholtz, Método dos Elementos de Contorno, Método dos Elementos Finitos. 


\section{Introdução}

Considere a Equação da Onda para problemas escalares em duas dimensões [1], dada por:

$$
U_{, i i}(\boldsymbol{X}, t)=\frac{1}{c^{2}} \ddot{U}(\boldsymbol{X}, t)
$$

Na Eq. (1), $\boldsymbol{X}$ significa a posição $\left(x_{1}, x_{2}\right)$ no espaço e o potencial $U(\boldsymbol{X}, t)$ pode representar um deslocamento, a dilatação volumétrica ou mesmo a pressão atuante no meio, de acordo a interpretação física do problema. No caso de problemas de acústica, o termo "c" significa a velocidade de propagação da onda num meio homogêneo. Tal equação pode ser deduzida pela simplificação dos termos relativos à Equação de Campo Escalar [2], apresentada na Eq. (2).

$$
\left(k_{i j} U_{, j}\right)_{, i}+\lambda_{1} \ddot{U}+\lambda_{2} \dot{U}+\lambda_{3} U+p=0
$$

O termo $p=p(\boldsymbol{X}, t)$ é uma função conhecida, distribuída no domínio. Na análise vibracional buscamse as configurações de equilíbrio associadas às frequências naturais. Assim, admitindo-se o movimento como sendo harmônico, chega-se a Equação de Helmholtz, dada pela seguinte equação:

$$
k u_{, i i}+\frac{\omega_{n}^{2}}{c^{2}} u=0
$$

Em que $\omega_{n}$ representa a frequência de excitação dinâmica e $u(\boldsymbol{X})$ a amplitude do potencial escalar. Este problema também configura o problema de autovalor [3] que será representado mais adiante, onde se procuram calcular as frequências naturais associadas, que são funções da rigidez e da massa. A solução analítica e numérica da Eq. (3) depende intrinsecamente das condições de fronteira, da geometria do domínio estabelecido, das suas propriedades físicas e da frequência imposta.

\section{Equações Integrais do MECID}

A abordagem habitual do MEC para o operador de Laplace é mantida, uma vez que se assume que tal estratégia resulta numa precisão bastante satisfatória. Assim, considera-se a Equação de Helmholtz, advinda da equação da onda, em sua forma integral inversa [4], conforme Eq. (4):

$$
\begin{aligned}
& \mathrm{c}(\xi) u(\xi)+\int_{\Gamma} \mathrm{u}(\mathrm{X}) q^{*}(\xi ; X) d \Gamma-\int_{\Gamma} \mathrm{q}(\mathrm{X}) u^{*}(\xi ; X) d \Gamma= \\
& \frac{\omega_{n}^{2}}{c^{2}} \int_{\Omega} u(\mathrm{X}) u^{*}(\xi ; X) d \Omega
\end{aligned}
$$

$\mathrm{Na}$ Eq.(4), $q(\boldsymbol{X})$ é a derivada normal do potencial; as funções auxiliares $u^{*}(\xi ; X)$ e $q^{*}(\xi ; X)$ são respectivamente a solução fundamental e sua derivada normal [4]. Devido o núcleo do lado direito da Eq. (4) conter valores de $u(\boldsymbol{X})$ no interior que não podem ser diretamente transferidos para o contorno, estes devem ser interpolados através de funções auxiliares, da classe das funções de base radial [5], na seguinte forma:

$$
u\left(X^{j}\right) u^{*}\left(\xi^{i} ; X^{j}\right)=F^{j}\left(X^{j} ; X\right)^{\xi} \alpha^{j}
$$

As funções $F^{j}\left(X^{j} ; X\right)$ são funções de base radial e ${ }^{\xi} \alpha^{j}$ os coeficientes a determinar. Assim, é possível usar a seguinte transformação matemática, que caracteriza o procedimento MECID [6]:

$\int_{\Omega}{ }^{\xi} \alpha^{j} F^{j}\left(X^{j} ; X\right) d \Omega=\int_{\Omega}{ }^{\xi} \alpha^{j} \Psi_{, i i}^{j}(\mathrm{X}) \mathrm{d} \Omega=$ $\int_{\Gamma}{ }^{\xi} \alpha^{j} \Psi_{, i}^{j}(X) n_{i}(X) \mathrm{d} \Gamma={ }^{\xi} \alpha^{j} \int_{\Gamma} \eta^{J}(\mathrm{X}) \mathrm{d} \Gamma(6)$

As funções $\Psi^{j}$ e $\eta^{J}$ são primitivas de $F^{j}\left(X^{j} ; X\right)$. Tal operação se faz necessária para construir a matriz de inércia $[\bar{M}]$, na seguinte forma:

$$
[\bar{H}]\{u\}=\frac{\omega_{n}^{2}}{c^{2}}[\bar{M}]\{u\}
$$

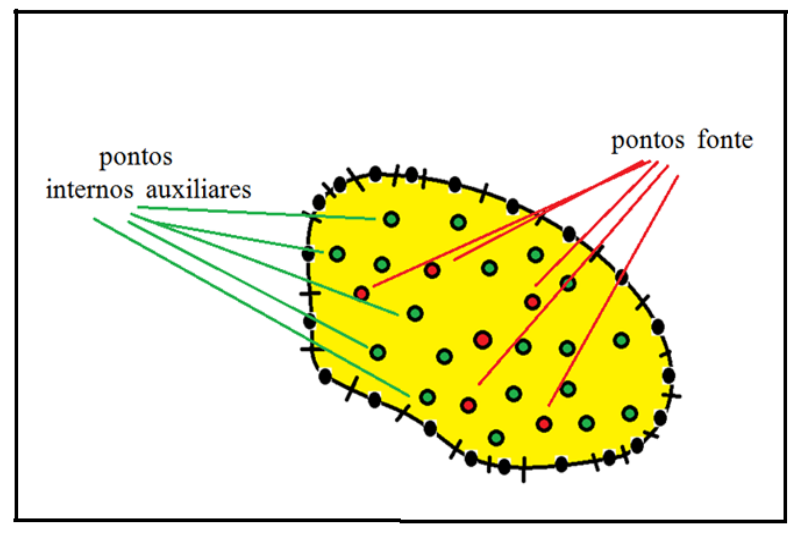

Figura 1: Posicionamento distinto dos pontos fonte $\xi$ e dos pontos interpolantes Xi situados no interior do domínio

Para melhorar o desempenho da MECID é preciso introduzir pontos internos interpolantes e também pontos fonte no interior do domínio, estes últimos, fornecem os graus de liberdade necessários para uma simulação dinâmica satisfatória. Estes pontos não podem ser coincidentes, conforme mostra a Fig. (1). Maiores detalhes podem ser colhidos na referência [7]. Deste modo, têm-se a expressão MECID que permite calcular não só a amplitude $u(\boldsymbol{X})$ em cada ponto, como também as frequências naturais ou autovalores $\omega_{n}$ do sistema no caso dos problemas de vibração livre, dados por:

$$
[\bar{H}]-\frac{\omega_{n}^{2}}{c^{2}}[\bar{M}]=0
$$




\section{Equações Integrais do MEF}

O MEF, diferentemente do MECID, baseia-se na discretização do domínio global, através de subdomínios locais $\delta \Omega$. A equação integral a ser utilizada apresenta-se na forma fraca [8], obtida através da utilização da função auxiliar $v(X)$ e suas propriedades, conforme Eq. (9).

$$
\begin{gathered}
\int_{\Omega} u(X)_{, i} v(X)_{, i} d \Omega=\int_{\Gamma} h(X) v(X) d \Gamma- \\
\frac{\omega_{n}^{2}}{c^{2}} \int_{\Omega} u(X) v(X) d \Omega
\end{gathered}
$$

Onde $h(X)$ representa o valor da derivada normal do potencial ou condição de Neumann, caso esteja prescrita. Inicialmente, o MEF trabalha de forma local, mas compõe matrizes globais válidas em todo 0 domínio, gerando assim um sistema matricial linear com solução única, após imposição das condições essenciais e naturais.

A malha utilizada para a aplicação do MEF é do tipo estruturada com elementos triangulares, utilizando uma função polinomial de primeiro grau para representar a função auxiliar $v(X)$ [8], conforme expressa a Eq. (10):

$$
v\left(x_{1}, x_{2}\right)=a_{i}+b_{i} x_{1}+c_{i} x_{2}
$$

Nesta última equação $v\left(x_{1}, x_{2}\right)$ assume valores nulos nas interseções vizinhas e valor unitário no ponto prescrito ou ponto a ser calculado no elemento finito triangular durante a interpolação no elemento [8].

\section{Resultados e Discussões}

O primeiro exemplo analisado consiste na aplicação da Equação de Helmholtz em um problema bidimensional com solução harmônica, conforme apresentado pela Eq. (11). As características do problema podem ser observadas na Fig. (2):

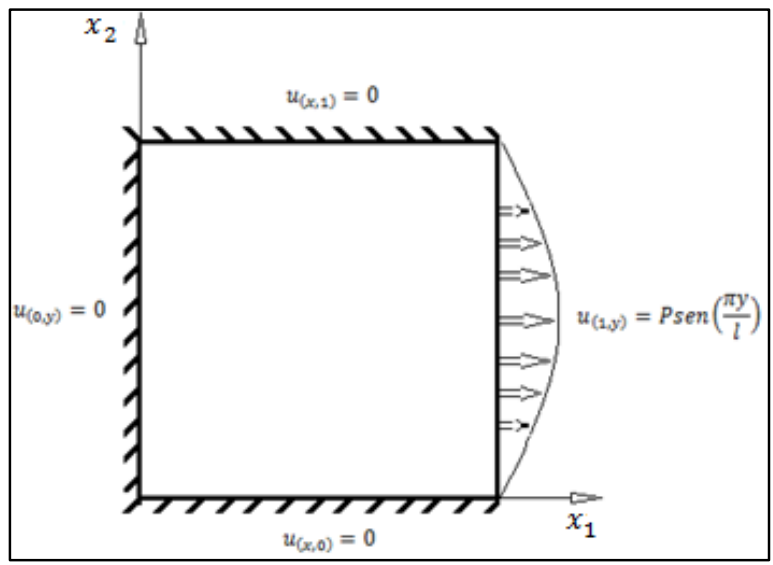

Figura 2: Membrana apresentando as condições de contorno e a localização do sistema de coordenadas.

$$
k\left[\left(\frac{\partial^{2} u}{\partial x_{1}^{2}}\right)+\left(\frac{\partial^{2} u}{\partial x_{2}^{2}}\right)\right]=-w^{2} u
$$

A solução analítica para o problema é dada por:

$$
u_{\left(x_{1}, x_{2}\right)}=\frac{\operatorname{sen}\left(x_{1} \sqrt{w^{2}-\pi^{2}}\right) \operatorname{sen}\left(\frac{\pi x_{2}}{l}\right)}{\operatorname{sen}\left(\sqrt{w^{2}-\pi^{2}}\right)}
$$

Por questão de conveniência, fixou-se $w=\frac{3 \pi}{2} \mathrm{~Hz}$, obtendo assim as soluções numéricas pelo MECID e MEF sem influência do fenômeno da ressonância.

Têm-se na Fig. (3), as curvas de erro médio percentual MECID, onde para duas malhas com diferentes níveis de refinamento $\mathrm{Pn}, \mathrm{Pf}$ e $\mathrm{Pi}$ representam respectivamente as quantidades de pontos nodais de contorno (com nós duplos nos cantos), os pontos fonte e pontos internos interpolantes.

A curva de erro médio percentual MEF conforme apresentada pela Fig. (4), foi obtida utilizando uma malha estruturada triangular, variando o refinamento de 62 a 5000 elementos, onde no seu refinamento máximo consta um total de 2601 pontos, sendo 200 pontos no contorno e 2401 pontos internos.

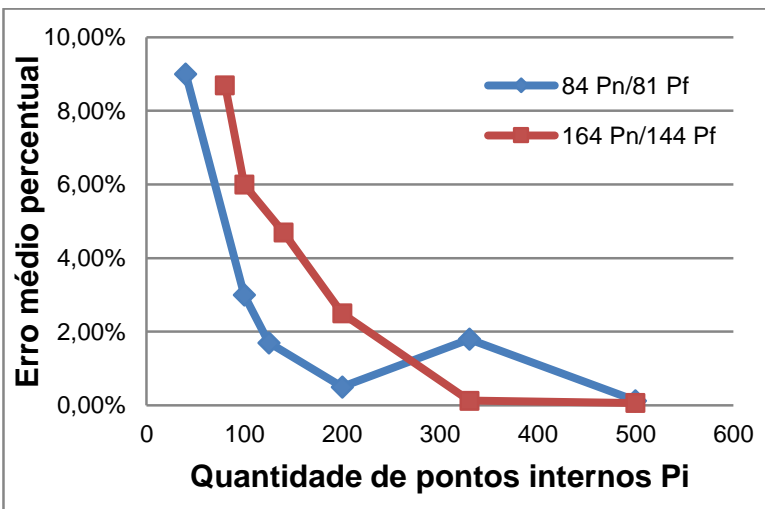

Figura 3:Representação dos erros para diferentes refinamentos, tomando como unitário o valor da frequência, utilizando o MECID. 164

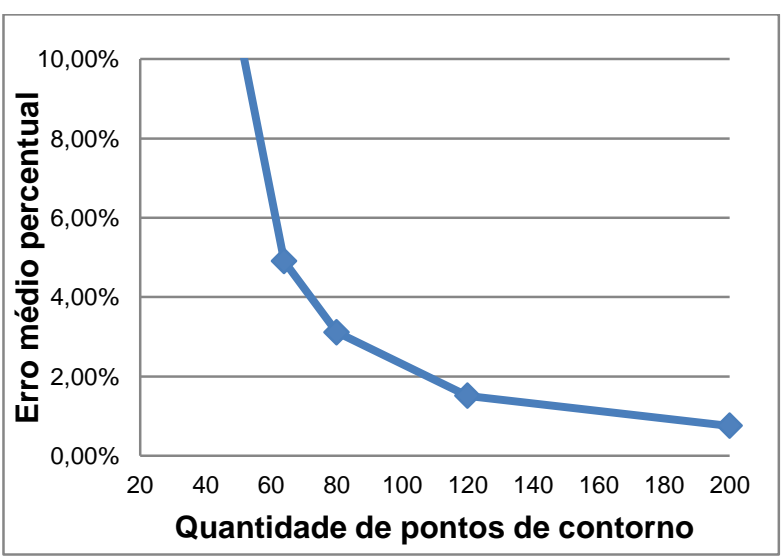

Figura 4:Curva de erro médio percentual com o refinamento de malha apresentado pelo MEF.

Pode-se observar um bom desempenho MECID para a malha de $164 \mathrm{Pn} / 144 \mathrm{Pf}$ em relação aos resultados do $\mathrm{MEF}$, mesmo para o maior refinamento apresentado de 5000 elementos finitos em malha estruturada, isto demonstra a adequação do MECID neste tipo de aplicação. 
O segundo exemplo apresentado consiste num problema de autovalor, onde se buscam os valores das frequências naturais para uma dada condição de contorno. No caso, analisa-se uma membrana quadrada de dimensões unitárias, mostrada na Fig. (5).

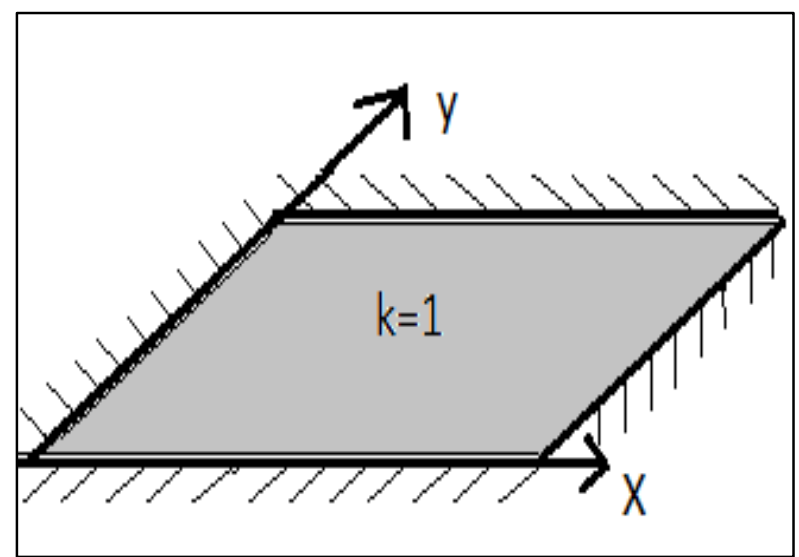

Figura 5: Membrana apresentando as condições de contorno, a localização do sistema de coordenadas e o valor da constante de rigidez $\mathrm{k}$.

Os valores analíticos [9] para frequências naturais são definidos conforme a expressão (13).

$$
\omega_{m n}=\frac{\pi \sqrt{m+n}}{L}, L=1
$$

Tabela 1: Autovalores obtidos pelo MECID para as variações de $\mathrm{m}$ e $\mathrm{n}$, utilizando 164 Pontos nodais com nó duplo e 144 pontos internos fonte.

\section{FREQUÊNCIA}

\begin{tabular}{|c|c|c|c|c|}
\hline \multicolumn{2}{|c|}{ Combinação $w_{m n}$} & \multirow[t]{2}{*}{ Analítica } & \multirow[t]{2}{*}{ *104 Pi } & \multirow[t]{2}{*}{ *484 Pi } \\
\hline $\mathbf{M}$ & $\mathbf{N}$ & & & \\
\hline 1 & 1 & 4,4428 & 4,4037 & 4,4468 \\
\hline 1 & 2 & 7,0248 & 6,7926 & 7,0249 \\
\hline 2 & 1 & 7,0248 & 6,8481 & 7,0249 \\
\hline 2 & 2 & 8,8858 & 8,2799 & 8,8641 \\
\hline 1 & 3 & 9,9346 & 9,3685 & 9,9451 \\
\hline 3 & 1 & 9,9346 & 9,5649 & 9,9671 \\
\hline 2 & 3 & 11,3272 & 10,2531 & 11,3313 \\
\hline 3 & 2 & 11,3272 & 10,3719 & 11,3310 \\
\hline 1 & 4 & 12,9531 & 10,5117 & 13,0178 \\
\hline 4 & 1 & 12,9531 & 10,9089 & 13,3982 \\
\hline
\end{tabular}

* Pontos internos interpolantes

Com os dados analíticos apresentados pela Tab. (1), já se pode observar uma boa convergência do MECID para os primeiros valores de frequência natural, obtidos através da malha com menor refinamento (104 pontos internos distribuídos), garantindo desde já uma boa confiabilidade para o método.

Tabela 2: Autovalores obtidos pelo MEF para as variações de $\mathrm{m}$ e $\mathrm{n}$.

\begin{tabular}{|c|c|c|c|c|}
\hline \multicolumn{2}{|c|}{ FREQUÊNCIA } & \multirow{3}{*}{ Analítica } & \multirow{3}{*}{$\begin{array}{c}{ }^{*} 1800 \mathrm{el} \\
841 \mathrm{pi} \\
120 \mathrm{pc}\end{array}$} & \multirow{3}{*}{$\begin{array}{c}{ }^{*} 3200 \mathrm{el} \\
1521 \mathrm{pi} \\
160 \mathrm{pc}\end{array}$} \\
\hline \multicolumn{2}{|c|}{ Combinação $w_{m n}$} & & & \\
\hline $\mathbf{M}$ & $\mathbf{N}$ & & & \\
\hline 1 & 1 & 4,4428 & 4,4511 & 4,4485 \\
\hline 1 & 2 & 7,0248 & 7,0448 & 7,0376 \\
\hline 2 & 1 & 7,0248 & 7,0541 & 7,0428 \\
\hline 2 & 2 & 8,8858 & 8,9385 & 8,9174 \\
\hline 1 & 3 & 9,9346 & 9,9928 & 9,9669 \\
\hline 3 & 1 & 9,9346 & 9,9931 & 9,9696 \\
\hline 2 & 3 & 11,3272 & 11,4038 & 11,3729 \\
\hline 3 & 2 & 11,3272 & 11,4524 & 11,4001 \\
\hline 1 & 4 & 12,9531 & 13,0697 & 13,0215 \\
\hline 4 & 1 & 12,9531 & 13,0725 & 13,0230 \\
\hline
\end{tabular}

* el, pi e pc corresponde ao número de elementos, pontos internos e pontos no contorno respectivamente

Pela Tab. (2), também é possível observar uma boa convergência para o MEF, indicando uma concordância entre os valores obtidos numericamente e os valores analíticos, para ambos os métodos, demonstrando assim, a precisão e adequação das duas técnicas neste tipo de problema. Cabe destacar, entretanto, o bom desempenho do MECID, que utiliza um procedimento matemático aproximado para a representação da inércia do sistema, através de funções de base radial. Também empregou um número menor de graus de liberdade do que o MEF, alcançando uma precisão similar para o cálculo de um determinado número de frequências mais baixas.

\section{Conclusões}

O condicionamento da realização de numerosos testes, em diferentes classes de problemas afins, tem como objetivo a busca pela precisão e confiabilidade das inovações aplicadas ao método analisado. Os resultados aqui apresentados mostraram-se satisfatórios ao MECID, uma vez que este necessitou de um menor refinamento de malha, apresentando bons resultados conforme os dados obtidos. O bom desempenho do MECID deve-se principalmente a dois fatores. Primeiramente, é devido ao fato de que o operador de Laplaciano é transformado matematicamente em termos integrais inversas de acordo com os procedimentos típicos do MEC, que são reconhecidamente eficientes. Em segundo lugar, o procedimento MECID é similar a um processo de interpolação através de funções radiais, 
que são mais simples e eficazes, apresentando ótima precisão, como atestam as simulações realizadas na representação de imagens, tabelas e cálculo de superfícies.

\section{Agradecimentos}

A CAPES por patrocinar este trabalho e a Universidade Federal do Espírito Santo pelo apoio a pesquisa.

\section{Referências}

[1] GRAFF, K.F., Wave Motion in Elastic Solids, Dover Publications, New York, 1975.

[2] LOEFFLER NETO, C. F., Modelos Mecânicos Derivados da Educação de Campo Escalar Generalizada. Revista Militar de Ciência e Tecnologia, Vol. 10, 24-38, (1992).

[3] HADLEY, G. Linear Algebra. USA: Addison Wesley Publishing Company, 1972.

[4] C. A. Brebbia, J. C. F. Telles, L.C. Wrobel, Boundary Element Techniques, Springer Verlag, first ed., Berlin Heidelberg, 1984.
[5] M. D. Buhmann, Radial Basis Functions: Theory and Implementations, first ed., New York, Cambridge University Press, 2003.

[6] BARCELOS, H. M. Comparação de Desempenho entre a Formulação Direta do Método dos Elementos de Contorno com Funções Radiais e o Método dos Elementos Finitos em Problemas de Poisson e Helmholtz 117f. Dissertação (Mestrado em Engenharia Mecânica), Programa de Pós-Graduação em Engenharia Mecânica, Departamento de Engenharia Mecânica, Universidade Federal do Espírito Santo, Espírito Santo, 2014.

[7] LOEFFleR NeTO, C. F.; PEREIRA, P. V. M.; BARCELOS, H. M., Direct Interpolation Technique using Radial Basis Functions Applied to the Helmholtz Problem. In: International Conference on Boundary Element and Meshless Techniques, 2014, Florence. International Conference on Boundary Element and Meshless Techniques XV BeTeq 2014, v. 1. p. 314-320, 2014.

[8] RINCON, M. A.; LIU, I.S. Liu, Introdução ao Método dos Elementos Finitos, Computação e Análise em Equações Diferenciais Parciais, UFRJ, Cap. 4, 3를 Ed, 2010. 2014.

[9] MEIROVITCH, L. Analytical Methods in Vibration: London, The Macmillan Company, 1967 